\title{
Discriminating the heavy jet production mechanisms in associated $Z$ + heavy flavor events at the LHC
}

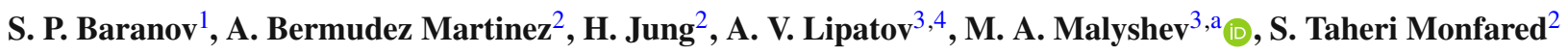 \\ ${ }^{1}$ Lebedev Physics Institute, Moscow, Russia \\ ${ }^{2}$ Deutsches Elektronen-Synchrotron DESY, Hamburg, Germany \\ ${ }^{3}$ Skobeltsyn Institute of Nuclear Physics, Lomonosov Moscow State University, Moscow, Russia \\ ${ }^{4}$ Joint Institute for Nuclear Research, 141980 Dubna, Moscow region, Russia
}

Received: 13 November 2021 / Accepted: 7 February 2022 / Published online: 18 February 2022

(C) The Author(s) 2022

\begin{abstract}
We reconsider the associated $Z$ boson and charm or beauty jet production at the LHC by paying special attention to the formation dynamics of heavy jets. Two different approaches are studied: first one, where heavy quarks are produced in the hard scattering subprocesses, implemented in the Monte-Carlo generator PEGASUS, and another method, where the hard scattering is calculated at NLO with MADGRAPH5_AMC@NLO and TMD parton shower is included (implemented in the Monte-Carlo generator CASCADE3). We compare the predictions obtained in both schemes with latest experimental data for associated $Z+b$ production cross sections and the relative production rate $\sigma(Z+c) / \sigma(Z+b)$ collected by the ATLAS and CMS Collaborations at $\sqrt{s}=$ $13 \mathrm{TeV}$. We introduce two kinematic observables (denoted as $z_{b}$ and $p_{T}^{\text {rel }}$ ) which can be used to discriminate between the heavy jet production mechanisms. Using these variables we trace the shape of the simulated $b$-jet events and recommend that these observables be taken into consideration in the forthcoming experimental analyses.
\end{abstract}

\section{Motivation}

With the LHC in operation, one can access a number of 'rare' processes which could have never been systematically studied at previous accelerators. In this article we revisit the associated production of $Z$ bosons and heavy quark jets. This process involves both weak and strong interactions and therefore serves as a complex test of the Standard Model, perturbative QCD and our knowledge of parton densities. On the experimental side, we have at our disposal the data collected by ATLAS and CMS collaborations [1-5].

\footnotetext{
a e-mail: malyshev@theory.sinp.msu.ru (corresponding author)
}

Earlier, we have demonstrated $[6,7]$ a quite reasonable agreement between the theoretical and experimental results with respect to many observables, such as the differential cross sections and particle correlations. Here we wish to go one step deeper in our understanding and draw attention to two new observables which can be used as clean probes of $b$-jet formation dynamics. We aim at a difference between 'prompt' and 'non-prompt' production cases. The former class refers to the situation when the $b$-quark is produced in the hard scattering subprocess; it further radiates lighter partons and after all evolves into a jet containing $b$-hadrons. The latter class refers to jets initiated by a light parton (gluon or quark), and then $b$-quarks (or rather $b$-hadrons) appear from a parton evolution cascade. We discuss kinematic criteria that can be helpful to discriminate these cases. Namely, we find that in the jets originating from $b$-quarks (i.e., 'prompt'), $b$-hadrons carry larger momentum fraction $z_{b}$ than in other ('non-prompt') jets. Second, in the prompt jets, $b$-hadrons move closer to the jet axis. The goal of the study is to give quantitative estimates and to see to what extent our expectations are supported by real data.

\section{Theoretical framework}

There are two commonly used approaches in perturbative QCD (pQCD) calculations for cross sections of processes containing heavy quarks. One of these approaches is the so-called four-flavour number scheme (4FNS), where only gluon distributions and first two quark generations are involved in the QCD evolution equations for parton (quark and gluon) densities in a proton, so that $b$-quarks appear in a massive final state as a result of gluon splitting $g \rightarrow b \bar{b}$ at leading order. The second approach is the five-flavour number scheme (5FNS), which allows a $b$-quark density in the 
initial state where the $b$-quark is typically treated massless above the flavour threshold. Up to all orders, the 4FNS and 5FNS schemes should give exactly the same results (up to the power corrections $\left.\sim m_{b}^{2} / \mu^{2}\right)$, while at a given order differences can occur (see Ref. [8] for an review). ${ }^{1}$ In the calculations below, we apply the 5FNS schemes for MADGRAPH5_AMC@NLO with CASCADE3.

In another framework, based on the CCFM evolution equation, only the gluon density but no heavy quark (and no light sea-quarks) parton densities exist. In the approach used with PEGASUS we apply a mixed scheme, using the gluon density from CCFM together with partons obtained in a DGLAP scheme with 5 active flavors (5FNS).

\subsection{Calculations with Monte-Carlo generator PEGASUS}

To calculate the $Z+b$-jet total and differential cross sections at the LHC we employ two different schemes based on the transverse momentum dependent (TMD) quark and gluon distributions in a proton. ${ }^{2}$ The first method was proposed in [6] and relies mainly on the $\mathcal{O}\left(\alpha \alpha_{s}^{2}\right)$ off-shell gluon-gluon fusion subprocess:

$g^{*}+g^{*} \rightarrow Z+Q+\bar{Q}$,

which gives the leading contribution to the production cross section in the small $x$ region, where the gluon density dominates over the quark densities. An essential point here is using the CCFM evolution equation to describe the QCD evolution of the TMD gluon density in a proton. The gauge-invariant off-shell amplitude for the gluon-gluon fusion subprocess has been calculated in Ref. $[13,14]$, where all the relevant technical details are explained.

In addition, we take into account two subleading subprocesses involving quarks in the initial state. These are the flavor excitation processes

$q+Q \rightarrow Z+Q+q$,

and the quark-antiquark annihilation processes

$q+\bar{q} \rightarrow Z+Q+\bar{Q}$,

which could play a role essentially at large transverse momenta (or, respectively, at large $x$ which is needed to produce large $p_{T}$ events) where the quarks are less suppressed or can even dominate over the gluon density. The contributions from the quark-induced subprocesses (2) and (3) are calculated within a conventional DGLAP-based (collinear) factorization scheme, which provides better theoretical grounds

\footnotetext{
1 The consistency of both approaches within the context of Parton Branching (PB) approach $[9,10]$ has been recently discussed [11].

${ }^{2}$ For detailed description and discussion of the different approaches involving TMD parton densities see, for example, review [12].
}

in the region of large $x$. The evaluation of the production amplitudes is straightforward and needs no explanations.

Our scheme $[6,7]$ represents a combination of two techniques with each of them being used at the kinematic conditions where it is best suitable. This scheme is implemented in the Monte-Carlo event generator PEGASUS [15], which has been used for numerical calculations. Taking all the three subprocesses (1), (2) and (3) into account we extend the predictions to the whole kinematic range. Note that at least one heavy quark $Q$ is always present in the final state already at the amplitude level.

The parton-level calculation returned by PEGASUS has further been improved by including the effects of the initial and final state parton showering. For the collinear part of the calculation, that has been done using the conventional PYTHIA8 [16] algorithm. ${ }^{3}$ The off-shell part of the calculations includes this kind of correction in the form of TMD gluon densities. The subsequent decay $Z \rightarrow l^{+} l^{-}$(including the $Z / \gamma^{*}$ interference effects) is incorporated already at the production step at the amplitude level in order to fully reproduce the experimental setup.

For the TMD gluon density in a proton, we used a numerical solution of the CCFM equation [18-21]. We find it to be a suitable option since it smoothly interpolates between the small- $x$ Balitsky-Fadin-Kuraev-Lipatov (BFKL) [2224] gluon dynamics and large- $x$ DGLAP one. We adopted the latest JH-2013 parametrization, namely, we choose the JH-2013 set 2 [25]. The corresponding TMD gluon density was fitted to high-precision DIS data on the proton structure functions $F_{2}\left(x, Q^{2}\right)$ and $F_{2}^{c}\left(x, Q^{2}\right)$. The fit was based on TMD matrix elements and involves the two-loop strong coupling constant, the kinematic consistency constraint [26,27] and non-singular terms in the CCFM gluon splitting function [28]. For the conventional quark and gluon densities, we used the NNPDF 4.0 (LO) [29] set.

\subsection{Calculations with Monte-Carlo generator CASCADE3}

This method represents a more rigorous scheme based on the parton branching (PB) approach, which was introduced $[9,10]$ to treat the DGLAP evolution [30-33]. The method provides a solution of these equations and coincides with the standard methods to solve the DGLAP equations for inclusive distributions at NLO and NNLO. It allows one to simultaneously take into account soft-gluon emission at $z \rightarrow 1$ and the transverse momentum $q_{T}$ recoils in the parton branchings along the QCD cascade. The latter leads to a natural determination of the TMD quark and gluon densities in a proton. One of the advantages of this approach is that the PB TMDs

\footnotetext{
${ }^{3}$ In fact, we took the TMD parton shower tool implemented into the Monte Carlo event generator CASCADE3 [17] and applied it to the offshell gluon-gluon fusion subprocess (1).
} 
can be combined with standard (on-shell) production amplitudes, which can be calculated at higher orders with. Here we use matrix elements calculated with next-to-leading (NLO) order with MADGRAPH5_AMC@NLO [34] using the HERWIG6 subtraction terms, which are suitable for combination with PB-TMDs.

The tool to be used to calculate the observables within the PB approach is the Monte-Carlo event generator CASCADE3 [17]. A special procedure is adopted for the initial partons' transverse momenta: a transverse momentum is assigned according to the TMD density, and then the partonparton system is boosted to its center-of-mass frame and rotated in such a way that only the longitudinal and energy components are nonzero. The energy and longitudinal component of the initial momenta are recalculated taking into account the virtual masses $[17,35]$. This method keeps the parton-parton invariant mass exactly conserved, while the rapidity of the partonic system is approximately restored, depending on the transverse momenta.

The PB TMD parton densities were obtained from HERA I+II DIS data (details are described in [36]). In the numerical calculations below we use the PB-NLO-HERAI+II-2018 set 2 available in [37].

\section{Numerical results}

Before we turn to the discussion on the 'prompt' and 'nonprompt' $b$-jets we have to justify our approach by comparing the results of our simulations with the newest CMS and ATLAS data. We start by listing the parameters of our calculations. Following [38], we set the charm and beauty quark masses to $m_{c}=1.4 \mathrm{GeV}$ and $m_{b}=4.75 \mathrm{GeV}$, the mass of $Z$ boson $m_{Z}=91.1876 \mathrm{GeV}$, its total decay width $\Gamma_{Z}=2.4952 \mathrm{GeV}$, and $\sin ^{2} \theta_{W}=0.23122$. The electromagnetic coupling is taken as $\alpha\left(m_{Z}\right)=1 / 128.74$.

In the calculations performed with PEGASUS we set $\Lambda_{\mathrm{QCD}}^{(4)}=200 \mathrm{MeV}$ and use two-loop QCD coupling according to [25]. The default renormalization scale was taken as $\mu_{R}^{2}=m_{Z}^{2}$, while the default factorization scale for the offshell gluon-gluon fusion subprocess was $\mu_{F}^{2}=\hat{s}+\mathbf{Q}_{T}^{2}$, where $\mathbf{Q}_{T}$ is the net transverse momentum of the initial offshell gluon pair. This choice is dictated mainly by the CCFM evolution algorithm (see [25] for more information). For the quark-induced subprocesses (2) and (3) we keep it equal to the renormalization scale, $\mu_{F}=\mu_{R}$. To estimate the theoretical uncertainties of our PEGASUS calculations for offshell gluon-gluon fusion subprocess (1) we use auxiliary '+' and '-' TMD gluon densities instead of the default TMD gluon distribution functions. These two sets refer to the varied hard scales in the strong coupling constant $\alpha_{s}$ in the off-shell amplitude: '+' stands for $2 \mu_{R}$, while '-' refers to $\mu_{R} / 2$ (see [25]). For the quark-induced subprocesses (2) and (3) we just vary the hard scale around its default value between halved and doubled magnitude, as usual.

For the PB calculation with MG5AMC+CA3(Z+1)NLO, we set $m_{c}=1.47 \mathrm{GeV}, m_{b}=4.75 \mathrm{GeV}, \alpha_{s}\left(m_{Z}^{2}\right)=$ 0.118 and $\mu_{R}=\mu_{F}=\frac{1}{2} \sum_{i} \sqrt{m_{i}^{2}+p_{t, i}^{2}}$, where the sum runs over all particles and parton in the matrix element. The hard process calculations are performed at NLO with MADGRAPH5_AMC@NLO [34] with HERWIG6 subtraction terms and the TMD parton shower is simulated with CASCADE3 [17]. The theoretical uncertainties are obtained by varying the scale of the hard process by a factor 2 up and down, provided by MADGRAPH5_AMC@NLO.

\section{$3.1 Z+$ heavy quark jet production}

Now we are in position to present our numerical results. First we discuss $Z+b$ production cross sections measured by the ATLAS Collaboration at $\sqrt{s}=13 \mathrm{TeV}$ [5]. The experimental setup was as following: the transverse momenta of the leptons from the $Z$ decay are required to be $p_{T}^{\text {lead }}>27$, with pseudorapidities of $\left|\eta^{l}\right|<2.5$ for muons or $\left|\eta^{l}\right|<2.47$ for electrons (excluding $1.37<\left|\eta^{l}\right|<1.52$ ). The leptons are isolated from the jets by $\Delta R<0.4$. The invariant mass of the reconstructed $Z$-boson has to be within $76<m^{l l}<106$. The jets are reconstructed in the anti- $k_{T}$ algorithm with radius $R_{\text {jet }}=0.4$ and should have $p_{T}^{\text {jet }}>20 \mathrm{GeV}$ and rapidity $|y|<2.5$.

Our numerical predictions are shown in Figs. 1 and 2 in comparison with the latest ATLAS data [5]. The shaded bands represent the theoretical uncertainties estimated as described above. In all figures we show predictions (as described above) obtained from MG5AMC+CA3(Z+1)NLO based on a NLO calculation of $Z+1$ jets with MADGRAPH5_AMC@NLO and TMD parton showers from CASCADE3 with and without hadronization, together with predictions obtained from PEGASUS with and without parton shower.

The MG5AMC+CA3(Z+1)NLO calculations describe well the ATLAS measurements of the $Z$ boson and the $b$ jet rapidity and transverse momenta spectra at low and moderate $p_{T}$ within the theoretical and experimental uncertainties. From the comparison of MG5AMC+CA3(Z+1)NLO with and without fragmentation, we estimate the fragmentation correction of $\sim 10 \%$ in the larger transverse momentum regions, while at small $p_{T}(Z)$ and small $p_{T}$ (bjet) the corrections are significantly larger. These corrections are coming from $b$-hadrons which are outside the jet with $R=0.4$. Due to missing higher order contributions in the calculation $(Z+1$ at NLO) there is a notable underestimate of the data at large transverse momenta, namely $p_{T} \gtrsim 200 \mathrm{GeV}$. These missing higher order contributions lead also to deviations at small $\Delta \phi(Z, b), \Delta R(Z, b)$ and at large $\Delta y(Z, b)$. 

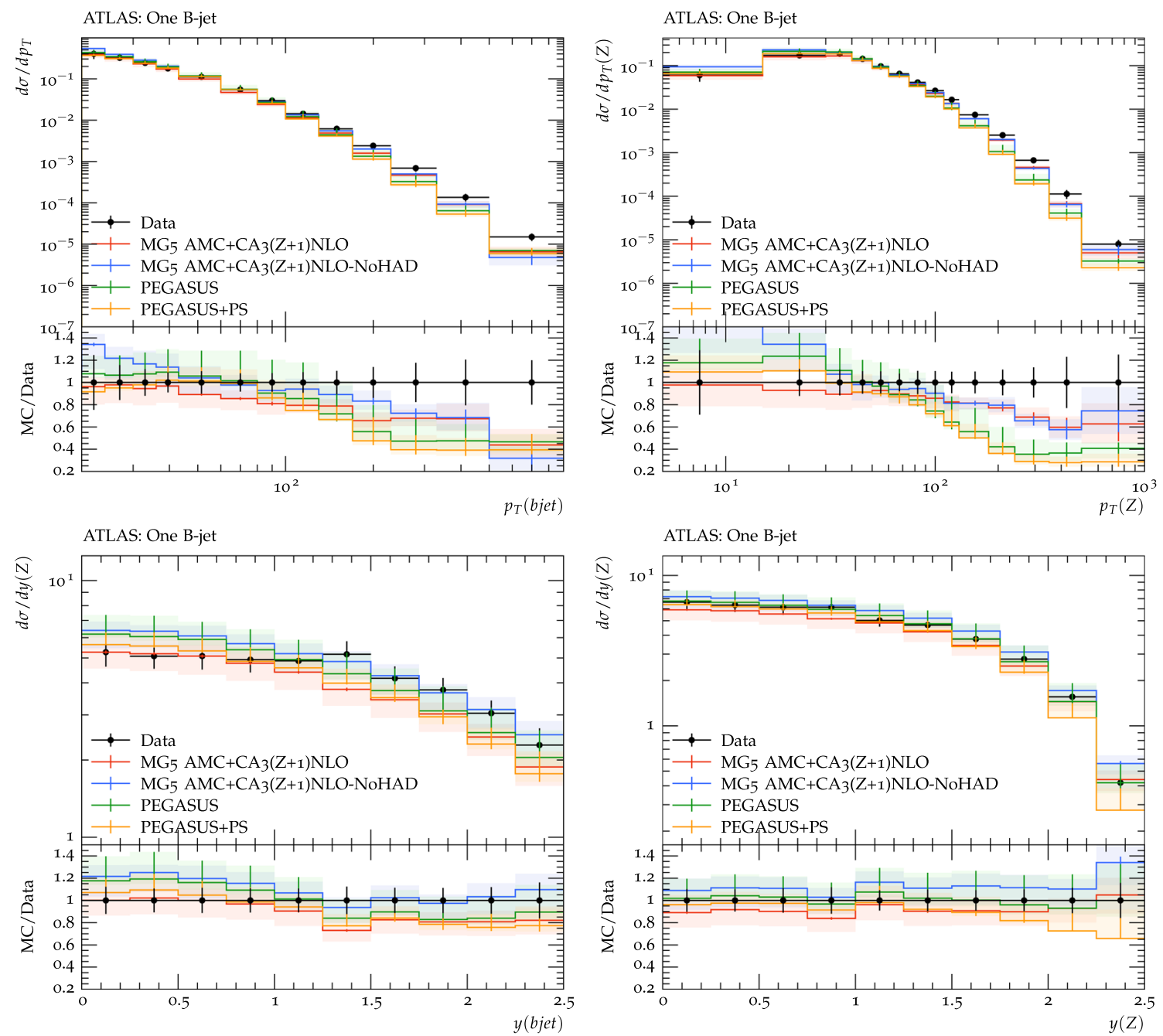

Fig. $1 Z+b$ production differential cross sections as functions of $b$-jet and Z-boson transverse momenta and rapidities at $\sqrt{s}=13$ TeV. ATLAS experimental data were taken from [5]

The PEGASUS predictions describe the data quite well within the estimated uncertainties, failing though at large $p_{T}$. The scenario implemented into PEGASUS, where the heavy quark is produced in the hard partonic scattering at the amplitude level, is able to reproduce well the measured distributions in $Z$ boson and $b$ jet rapidity and transverse momenta (at low and moderate $p_{T}$ ). It is interesting to observe, that the distribution of $\Delta \phi(Z, b)$ is well described, even at low $\Delta \phi(Z, b)$, which is in contrast with the PB result. One should however keep in mind that the PEGASUS calculations do not include fragmentation (unlike the PB ones). ${ }^{4}$ Taking into account the fragmentation effect may result in a $\sim 10 \%$ drop of cross section [39]. The inclusion of the corresponding fragmentation correction factor could simultaneously result in better agreement by the two approaches. In the $\Delta y(Z, b)$

\footnotetext{
$\overline{4}$ The same hadronization corrections as obtained from CASCADE3 apply also to PEGASUS.
}

we observe a similar behavior as for the PB predictions. The final state parton shower effects does not significantly affect the PEGASUS predictions. It can be easily understood since the main contributions here comes from initial state gluon radiation, which is already taken into account in the CCFMevolved gluon density.

Now we turn to associated $Z+c$ production at the LHC and discuss the relative production rate $\sigma(Z+c) / \sigma(Z+b)$ as measured by the CMS collaboration at $\sqrt{s}=13 \mathrm{TeV}$ [3]. The experimental cuts are: the leading lepton from the $Z$ decay is required to have a transverse momentum $p_{T}^{\text {lead }}>$ $26 \mathrm{GeV}$, while the subleading lepton must have $p_{T}^{\text {sublead }}>$ $10 \mathrm{GeV}$; with pseudorapidities within $\left|\eta^{l}\right|<2.4$ and the dilepton invariant mass should be $71<m^{l l}<111 \mathrm{GeV}$. The leptons are required to be isolated from the jets with $\Delta R<0.4$. The jets are required to have $p_{T}^{\text {jet }}>30 \mathrm{GeV}$ and $\left|\eta^{\mathrm{jet}}\right|<2.4$, reconstructed with the anti- $k_{T}$ algorithm with radius $R_{\text {jet }}=0.4$. 

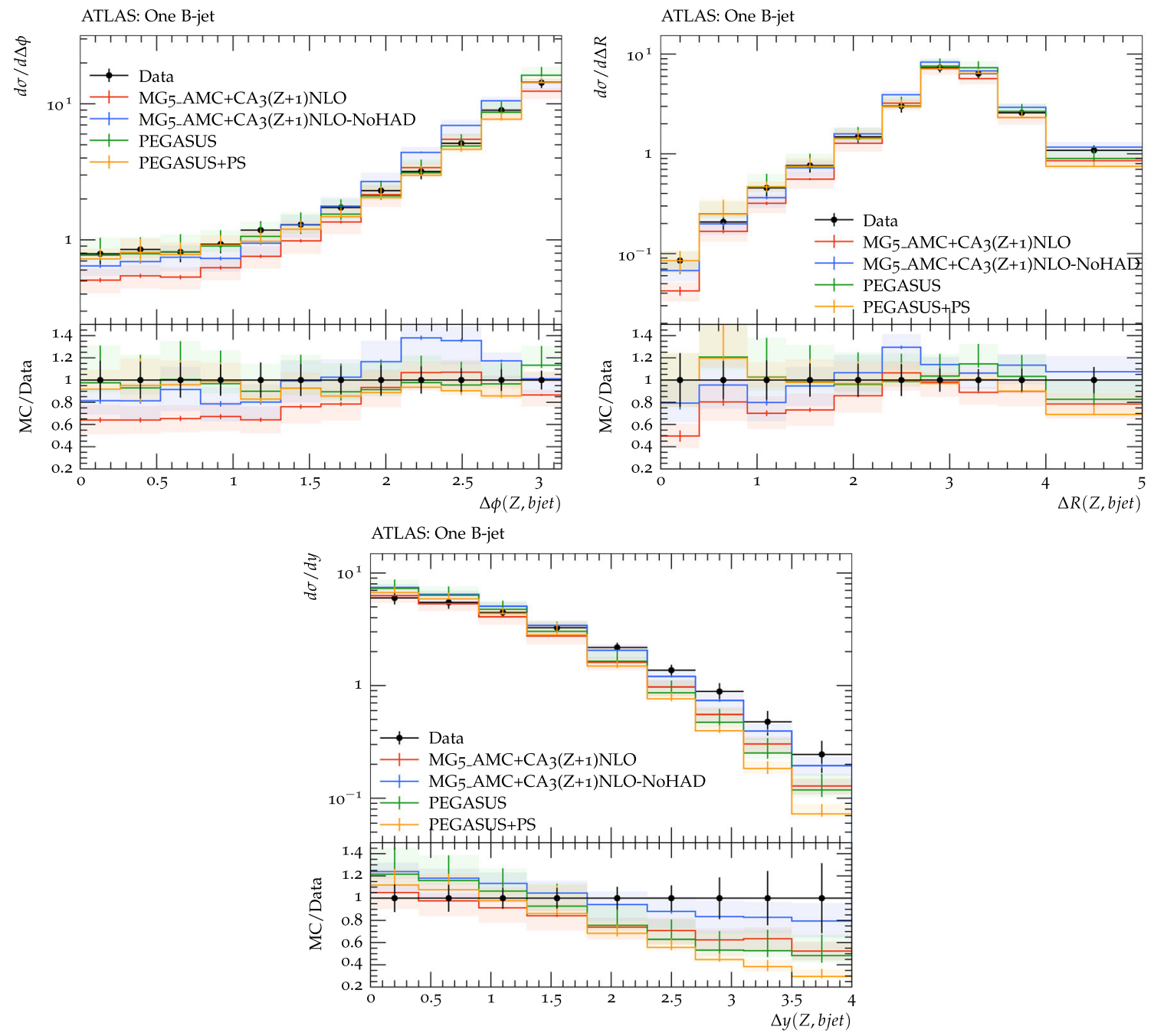

Fig. $2 Z+b$ production differential cross sections as functions of the azimuthal angle, angular difference and rapidity differences between $b$-jet and $Z$-boson at $\sqrt{s}=13 \mathrm{TeV}$. ATLAS experimental data were taken from [5]
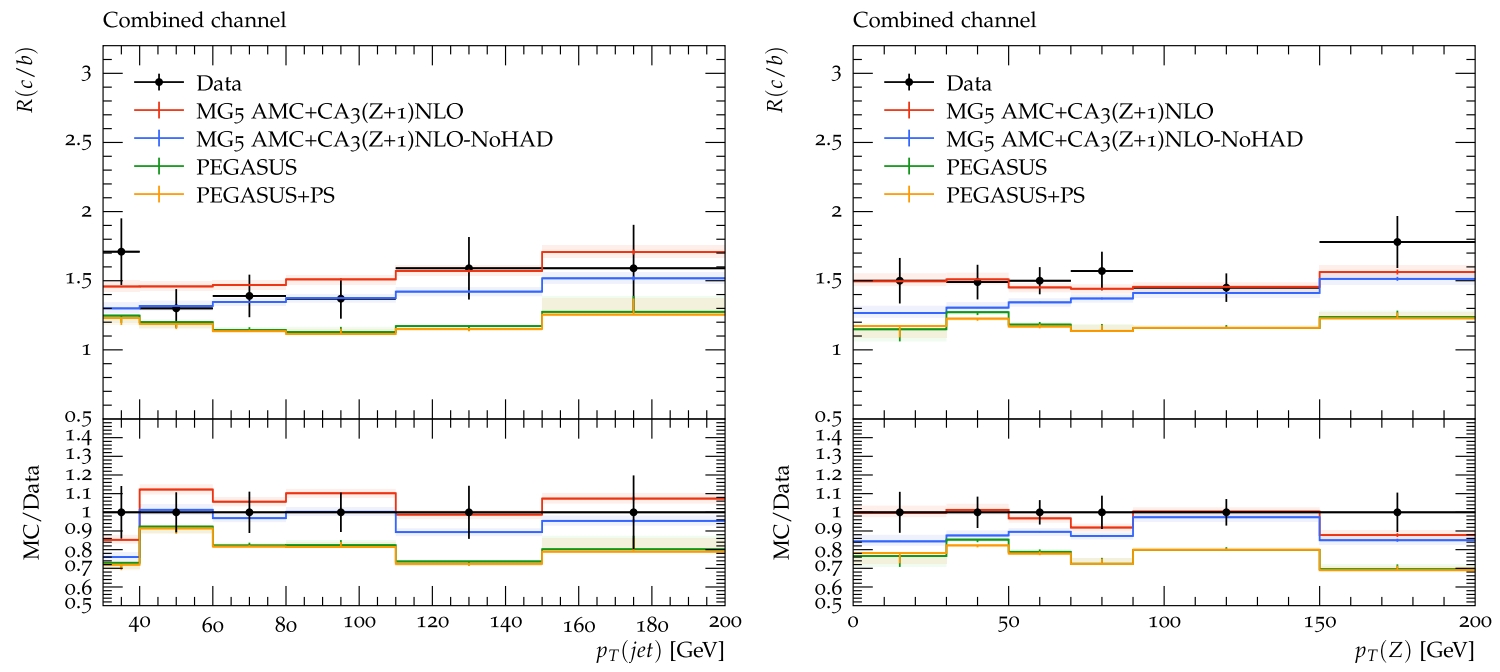

Fig. 3 The relative production rate $\sigma(Z+c) / \sigma(Z+b)$ as functions of heavy jet (left panel) and $Z$-boson (right panel) transverse momentum at $\sqrt{s}=13 \mathrm{TeV}$. The experimental data are from CMS [3] 

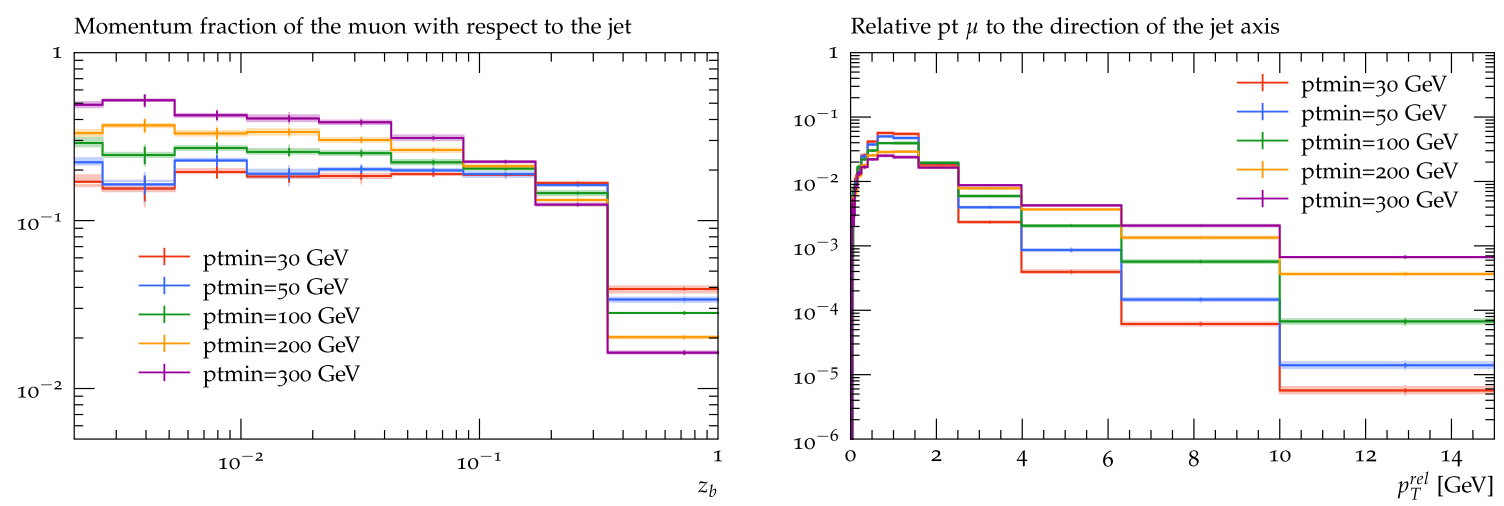

Fig. $4 Z+b$ production differential cross sections as functions of the energy fraction carried by the decay muon with respect to the total $b$-jet energy and the muon transverse momentum with respect to the $b$-jet axis at $\sqrt{s}=13 \mathrm{TeV}$ for different cuts on the minimal $p_{T}$ of the jet

In Fig. 3 we show the results of our calculations for the ratio $\sigma(Z+c) / \sigma(Z+b)$ as a function of $Z$ boson or jet transverse momenta in comparison with the CMS data [3]. The shaded bands represent the estimated uncertainties of our calculations. A good description of the CMS data can be obtained with MG5AMC+CA3(Z+1)NLO The predictions from PEGASUS lie below the data, though being compatible with the data at $\sim 2 \sigma$. Thus, despite the fact that both considered scenario provide a more or less reasonable description of inclusive production data (see Figs. 1 and 2), they are very distinct for $\sigma(Z+c) / \sigma(Z+b)$ observables and some correlation variables. The same conclusion was achieved earlier [7] when comparing the PEGASUS and SHERPA (NLO pQCD) predictions, where the same heavy jet production scenario, as in the MG5AMC+CA3(Z+1)NLO is employed. Below we will discuss the kinematic criteria that can be helpful in discriminating these two cases.

\subsection{Prompt and non-prompt $b$-jets}

In 5 FNS calculations often a $b$-quark is produced during the jet fragmentation process, in gluon splitting $g \rightarrow b \bar{b}$, while this contribution is small in the CCFM or 4FNS approaches, since a $b$-quark is already present in the hard process. It is therefore of great importance to find observables which could differentiate between a heavy-quark produced during the jet fragmentation fand the one present at the hard process.

Having demonstrated that the latest LHC data on $Z+b$ production can be well described by CASCADE and PEGASUS at least up to moderate transverse momenta, in the following we consider kinematic properties of $b$-jet production by investigating $B$-hadrons tagged via the semileptonic decay $B \rightarrow \mu+X$ : we consider the fractional energy $z_{b}$ carried by the decay muon with respect to the total $b$-jet energy and the muon transverse momentum $p_{T}^{\text {rel }}$ with respect to the $b$ jet axis. With both variables we aim to distinguish between prompt $b$-production, where the $b$-quark exists already at the matrix element level, and non-prompt $b$-production, where the $b$-quark is generated during the jet evolution. We expect large $z_{b}$ and small $p_{T}^{\text {rel }}$ (relative to the jet $p_{T}$ ) for prompt $b$ production, while non-prompt $b$-production would lead to significantly smaller values for $z_{b}$ and a larger tail for $p_{T}^{\text {rel }}$.

In Fig. 4 the distribution of $z_{b}$ and $p_{T}^{\text {rel }}$ are shown for different thresholds of the jet transverse momentum: $p_{T}^{\text {jets }}>30,50,100,200,300 \mathrm{GeV}$. Jets with larger transverse momenta provide larger phase space for parton radiation cascades. Accordingly, they show a larger fraction of 'non-prompt' $B$-hadrons resulting in a larger fraction of lowenergy muons (muons with low $z_{b}$ ) and in larger fraction of muons with high $p_{T}^{\text {rel }}$ (muons with large deviation from the jet direction).

We find, that the intuitive variables $z_{b}$ and $p_{T}^{\text {rel }}$ are very powerful to distinguish prompt and non-prompt $b$ production.

The observables $z_{b}$ and $p_{T}^{\text {rel }}$ are very well suited to discriminate between the heavy jet production mechanisms. An experimental measurement of these variables would be important to test the model predictions, and to confirm the importance of gluon splitting in the production of high $p_{T}$ $b$-jets.

\section{Conclusion}

We have considered the production of $Z$ bosons associated with charm and beauty jets at LHC conditions. We investigated two different schemes.

We find that the combination of three basic subprocesses (1)-(3) involving heavy quarks in final states provides a consistent description of $Z$ boson and/or $b$-jet transverse momenta and rapidity distributions as well as different correlation observables in $Z+b$ events at low and moderate $p_{T}$. This can be seen from a direct comparison between the 
model predictions obtained using the Monte-Carlo generator PEGASUS and recent LHC data.

In another approach we consider $Z+j e t$ production at NLO, where heavy quarks can be produced directly in the matrix element, or during the showering process. We perform the calculations based on the Parton Branching TMDs together with TMD shower for the initial state cascade. Such calculations were performed using MG5$\mathrm{AMC}+\mathrm{CA} 3(\mathrm{Z}+1) \mathrm{NLO}$. We find very good description of the measurement at not too large transverse momentum of the $Z$ boson.

We classify different $b$-jet production mechanisms as prompt and non-prompt, depending whether the heavy quark is present at matrix element level or generated during the jet evolution.

Events of the prompt and non-prompt types show rather different kinematic properties, that can be seen, in particular, in relative production rate $\sigma(Z+c) / \sigma(Z+b)$ measured very recently by the CMS Collaboration for the first time. The ratio of $c$ over $b$ jet production is also reasonably well described by the PB prediction of MG5AMC+CA3(Z+1)NLO.

Considering the $Z+b$ events as a representative example, we see that jets with larger transverse momenta contain larger fraction of non-prompt $b$-hadrons, that results in larger fraction of low-energy muons and in larger fraction of muons with large deviation from the jet direction. We recommend that the relevant observables, $z_{b}$ and $p_{T}^{\text {rel }}$, be used in the forthcoming experimental analyses.

Acknowledgements We thank G.I. Lykasov and S.M. Turchikhin for important comments and remarks. S.P.B., A.V.L. and M.A.M. are grateful to DESY Directorate for the support in the framework of Cooperation Agreement between MSU and DESY on phenomenology of the LHC processes and TMD parton densities. M.A.M. was also supported by the grant of the Foundation for the Advancement of Theoretical Physics and Mathematics "BASIS" 20-1-3-11-1. STM thanks the Humboldt Foundation for the Georg Forster research fellowship.

Data Availability Statement This manuscript has no associated data or the data will not be deposited. [Authors' comment: There are no external data associated with this manuscript.]

Open Access This article is licensed under a Creative Commons Attribution 4.0 International License, which permits use, sharing, adaptation, distribution and reproduction in any medium or format, as long as you give appropriate credit to the original author(s) and the source, provide a link to the Creative Commons licence, and indicate if changes were made. The images or other third party material in this article are included in the article's Creative Commons licence, unless indicated otherwise in a credit line to the material. If material is not included in the article's Creative Commons licence and your intended use is not permitted by statutory regulation or exceeds the permitted use, you will need to obtain permission directly from the copyright holder. To view a copy of this licence, visit http://creativecomm ons.org/licenses/by/4.0/.

Funded by SCOAP . $^{3}$.

\section{References}

1. C.M.S. Collaboration, JHEP 12, 039 (2013)

2. C.M.S. Collaboration, Eur. Phys. J. C 77, 751 (2017)

3. C.M.S. Collaboration, Phys. Rev. D 102, 032007 (2020)

4. ATLAS Collaboration, JHEP 10, 141 (2014)

5. ATLAS Collaboration, JHEP 07, 044 (2020)

6. S.P. Baranov, H. Jung, A.V. Lipatov, M.A. Malyshev, Eur. Phys. J. C 77, 772 (2017)

7. A.V. Lipatov, G.I. Lykasov, M.A. Malyshev, A.A. Prokhorov, S.M. Turchikhin, Phys. Rev. D 97, 114019 (2018)

8. F. Maltoni, G. Ridolfi, M. Ubiali, JHEP 07, 022 (2012)

9. F. Hautmann, H. Jung, A. Lelek, V. Radescu, R. Zlebcik, Phys. Lett. B 772, 446 (2017)

10. F. Hautmann, H. Jung, A. Lelek, V. Radescu, R. Zlebcik, JHEP 01, 070 (2018)

11. H. Jung, S. Taheri Monfared, arXiv:2106.09791 [hep-ph]

12. R. Angeles-Martinez et al., Acta Phys. Polon. 46, 2501 (2015)

13. S.P. Baranov, A.V. Lipatov, N.P. Zotov, Phys. Rev. D 78, 014025 (2008)

14. M. Deak, F. Schwennsen, JHEP 09, 035 (2008)

15. A.V. Lipatov, M.A. Malyshev, S.P. Baranov, Eur. Phys. J. C 80, 330 (2020)

16. T. Sjöstrand et al., Comput. Phys. Commun. 191, 159 (2015)

17. S. Baranov et al., Eur. Phys. J. C 81, 425 (2021)

18. M. Ciafaloni, Nucl. Phys. B 296, 49 (1988)

19. S. Catani, F. Fiorani, G. Marchesini, Phys. Lett. B 234, 339 (1990)

20. S. Catani, F. Fiorani, G. Marchesini, Nucl. Phys. B 336, 18 (1990)

21. G. Marchesini, Nucl. Phys. B 445, 49 (1995)

22. E.A. Kuraev, L.N. Lipatov, V.S. Fadin, Sov. Phys. JETP 44, 443 (1976)

23. E.A. Kuraev, L.N. Lipatov, V.S. Fadin, Sov. Phys. JETP 45, 199 (1977)

24. I.I. Balitsky, L.N. Lipatov, Sov. J. Nucl. Phys. 28, 822 (1978)

25. F. Hautmann, H. Jung, Nucl. Phys. B 883, 1 (2014)

26. J. Kwiecinski, A.D. Martin, P. Sutton, Z. Phys. C 71, 585 (1996)

27. B. Andersson, G. Gustafson, J. Samuelsson, Nucl. Phys. B 467, 443 (1996)

28. M. Hansson, H. Jung, arXiv:hep-ph/0309009

29. NNPDF Collaboration, arXiv:2109.02653

30. V.N. Gribov, L.N. Lipatov, Sov. J. Nucl. Phys. 15, 438 (1972)

31. L.N. Lipatov, Sov. J. Nucl. Phys. 20, 94 (1975)

32. G. Altarelli, G. Parisi, Nucl. Phys. B 126, 298 (1977)

33. Yu.L. Dokshitzer, Sov. Phys. JETP 46, 641 (1977)

34. J. Alwall et al., JHEP 07, 079 (2014)

35. M. Bengtsson, T. Sjostrand, M. van Zijl, Z. Phys. C 32, 67 (1986)

36. A. Bermudez Martinez et al., Phys. Rev. D 99, 074008 (2019)

37. N.A. Abdulov et al., Eur. Phys. J. C 81, 752 (2021)

38. Particle Data Group, PTEP 2020, 083 C01 (2020)

39. ATLAS Collaboration, Phys. Lett. B 706, 295 (2012) 